\title{
Application of matrix eigenvalue and eigenvector Jixia Deng
}

Marine college of Shandong jiaotong university, Weihai, Shandong,China

Keywords: matrix eigenvalue, differential equation, recurrence relation

Abstract. matrix characteristic value and are widely used in many fields, this paper mainly studies the the application of two aspects: the first is applied in solving problems for first order linear differential equations. The second is in the recursive relationship in the application.

\section{Introduction}

Matrix eigenvalue is the important content of higher mathematics. Simple solving matrix eigenvalue is a relatively easy thing to do, but applied to other field is not so simple, therefore, to inspire the the Matrix eigenvalue value for the application of interest. This paper through application examples let everybody eigenvalues.

\section{Application of eigenvalue in the solution of linear constant coefficient differential equations}

Proposition: First order linear differential equations with constant coefficients

$$
\left\{\begin{array}{c}
\frac{d y_{1}}{d t}=a_{11} y_{1}+a_{12} y_{2}+\ldots+a_{1 n} y_{n} \\
\frac{d y_{2}}{d t}=a_{21} y_{1}+a_{22} y_{2}+\ldots+a_{2 n} y_{n} \\
\mathrm{~L} \\
\frac{d y_{n}}{d t}=a_{n 1}+a_{n 2}+\ldots+a_{n n} y_{n}
\end{array}\right.
$$

Set $Y=\left(y_{1}, y_{2}, \ldots, y_{n}\right)^{T}, \frac{d Y}{d t}=\left(\frac{d y_{1}}{d t}, \frac{d y_{2}}{d t}, \ldots, \frac{d y_{n}}{d t}\right)^{T}$

$A=\left(a_{i j}\right)$ is the equations of coefficient matrix,

so the equations of writing matrix form is: $\frac{d Y}{d t}=A Y$

Set equations solutions is : $Y=e^{x} X$

That is $\left(\begin{array}{c}y_{1} \\ y_{2} \\ \mathrm{M} \\ y_{n}\end{array}\right)=e^{x}\left(\begin{array}{c}x_{1} \\ x_{2} \\ \mathrm{M} \\ x_{n}\end{array}\right)$

When matrix A diagonalization, $\lambda_{1}, \lambda_{2}, \cdots, \lambda_{n}$ is the matrix eigenvalue of $\mathrm{A}, \mathrm{n}$ linearly independent eigenvectors $X_{1}, X_{2}, \cdots, X_{n}, \quad e^{\lambda_{1} t} X_{1}, e^{\lambda_{2} t} X_{2}, \mathrm{~L}, e^{\lambda_{n} t} X_{n}$

Linear combination of them : $Y=c_{1} e^{\lambda_{1} t} X_{1}+c_{2} e^{\lambda_{2} t} X_{2}+\cdots+c_{n} e^{\lambda_{n} t} X_{n}$ is the solution of equations. Written in matrix form is: 


$$
Y=\left(X_{1}, X_{2}, \mathrm{~L}, X_{n}\right)\left(\begin{array}{llll}
e^{\lambda_{1} t} & & & \\
& e^{\lambda_{2} t} & & \\
& & O & \\
& & & e^{\lambda_{n} t}
\end{array}\right)\left(\begin{array}{c}
c_{1} \\
c_{2} \\
\mathrm{M} \\
c_{n}
\end{array}\right)
$$

Example:

To solve the differential equation $\left\{\begin{array}{l}\frac{\mathrm{d} x}{\mathrm{~d} t}=4 x-5 y, \\ \frac{\mathrm{d} y}{\mathrm{~d} t}=2 x-3 y,\end{array}(x(0), y(0))=(1,0)\right.$

Solution: $\boldsymbol{A}=\left(\begin{array}{ll}4 & -5 \\ 2 & -3\end{array}\right),|\lambda \boldsymbol{E}-\boldsymbol{A}|=\left|\begin{array}{cc}\lambda-4 & 5 \\ -2 & \lambda+3\end{array}\right|=(\lambda+1)(\lambda-2)$.

get eigenvalue of $\mathrm{A}$ is $\lambda_{1}=-1, \lambda_{2}=2$

$\lambda_{1}=-1$, the corresponding eigenvectors is $\left(\begin{array}{l}1 \\ 1\end{array}\right), \quad \lambda_{2}=2$, is $\left(\begin{array}{l}5 \\ 2\end{array}\right)$

Set $P=\left(\begin{array}{ll}1 & 5 \\ 1 & 2\end{array}\right) \quad \mathbf{X}=\left(\begin{array}{l}x \\ y\end{array}\right)$,

So there is $\left(\begin{array}{l}x \\ y\end{array}\right)=\left(\begin{array}{ll}1 & 5 \\ 1 & 2\end{array}\right)\left(\begin{array}{cc}e^{-t} & 0 \\ 0 & e^{2 t}\end{array}\right)\left(\begin{array}{l}c_{1} \\ c_{2}\end{array}\right)=\left(\begin{array}{l}c_{1} e^{-t}+5 c_{2} e^{2 t} \\ c_{1} e^{-t}+2 c_{2} e^{2 t}\end{array}\right)$

The $(x(0), y(0))=(1,0)$ into above, get $\left\{\begin{array}{l}c_{1}=-\frac{2}{3} \\ c_{2}=\frac{1}{3}\end{array}\right.$

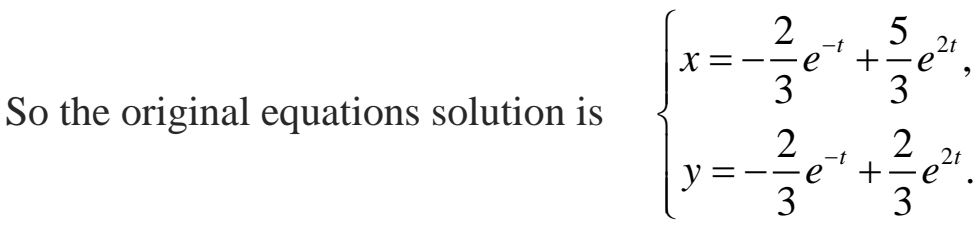

\section{Eigenvalues and eigenvectors in the application of linear recurrence relation}

Proposition : Set the $\mathrm{n}$ order linear cycle sequence $\left\{x_{n}\right\}$ meet the recursive relations $x_{n}=a_{1} x_{n-1}+a_{2} x_{n-2}+\mathrm{L}+a_{k} x_{n-k},(n=k+1, k+2, \mathrm{~L})$

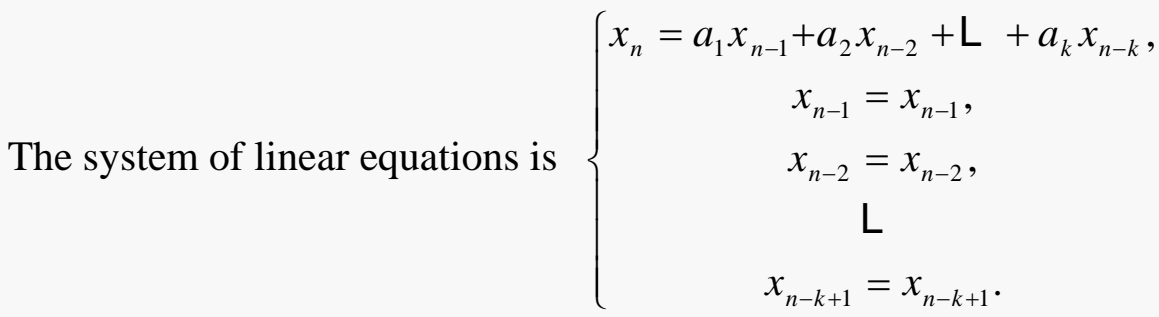

Using matrix form is $\left[\begin{array}{c}x_{n} \\ x_{n-1} \\ x_{n-2} \\ \mathrm{M} \\ x_{n-k+1}\end{array}\right]=\left[\begin{array}{ccccc}a_{1} & a_{2} & \mathrm{~L} & a_{k-1} & a_{k} \\ 1 & 0 & \mathrm{~L} & 0 & 0 \\ 0 & 1 & \mathrm{~L} & 0 & 0 \\ \mathrm{M} & \mathrm{M} & & \mathrm{M} & \mathrm{M} \\ 0 & 0 & \mathrm{~L} & 1 & 0\end{array}\right]\left[\begin{array}{c}x_{n-1} \\ x_{n-2} \\ x_{n-3} \\ \mathrm{M} \\ x_{n-k}\end{array}\right]$ 
Set $a_{n-k}=\left[\begin{array}{r}x_{n-1} \\ x_{n-2} \\ x_{n-3} \\ \mathrm{M} \\ x_{n-k}\end{array}\right], \quad A=\left[\begin{array}{ccccc}a_{1} & a_{2} & \mathrm{~L} & a_{k-1} & a_{k} \\ 1 & 0 & \mathrm{~L} & 0 & 0 \\ 0 & 1 & \mathrm{~L} & 0 & 0 \\ \mathrm{M} & \mathrm{M} & & \mathrm{M} & \mathrm{M} \\ 0 & 0 & \mathrm{~L} & 1 & 0\end{array}\right]$

So $a_{n-k+1}=A a_{n-k}, \quad a_{n-k+1}=A^{2} a_{n-k-1}=\mathrm{L}=A^{n-k} a_{1}$

among them $a_{1}=\left(x_{k}, x_{k-1}, \mathrm{~L}, x_{2}, x_{1}\right)^{T}$, the key is solve the $A^{n-k}$

Set $\mathrm{A}$ can diagonalization, there is invertible matrix $\mathrm{P}$, make $P^{-1} A P=B, \mathrm{~B}$ is the diagonal matrix, It is easy to get $A^{n-k}=P B^{n-k} P^{-1}$, so to write $x_{n}$

Example: The famous series :0,1,2,3,5,L $, F_{k}, \mathrm{~L}$, series has the condition $F_{0}=0, F_{1}=1, F_{k+2}=F_{k+1}+F_{k}$. To solve $F_{k}$

Soluation: set $\left\{\begin{array}{c}F_{k+2}=F_{k+1}+F_{k} \\ F_{k+1}=F_{k+1}\end{array}, \quad\right.$ matrix is $\quad\left(\begin{array}{l}F_{K+2} \\ F_{K+1}\end{array}\right)=\left(\begin{array}{ll}1 & 1 \\ 1 & 0\end{array}\right)\left(\begin{array}{l}F_{K+1} \\ F_{K}\end{array}\right)$

Set $A=\left(\begin{array}{ll}1 & 1 \\ 1 & 0\end{array}\right), \alpha_{k}=\left(\begin{array}{c}F_{k+1} \\ F_{k}\end{array}\right)$, so $\alpha_{0}=\left(\begin{array}{l}F_{1} \\ F_{0}\end{array}\right)=\left(\begin{array}{l}1 \\ 0\end{array}\right)$,

The equations simple form is $\quad \alpha_{k+1}=A \alpha_{k} \quad(k=1,2,3 \mathrm{~L})$.

$\alpha_{k+1}=A \alpha_{k}=A^{2} \alpha_{K-1}=A^{3} \alpha_{K-2}=\ldots \ldots=A^{K+1} \alpha_{0}$

$\alpha_{k}=A^{k} \alpha_{0} \quad(k=1,2,3 \mathrm{~L})$

To solve $A^{k}, \quad|\lambda E-A|=\left|\begin{array}{cc}\lambda-1 & -1 \\ -1 & \lambda\end{array}\right|=\lambda^{2}-\lambda-1$

Get eigenvalue of $A$ is $\lambda_{1}=\frac{1+\sqrt{5}}{2}, \lambda_{2}=\frac{1-\sqrt{5}}{2}$

eigenvector is $X_{1}=\left(\begin{array}{c}\lambda_{1} \\ 1\end{array}\right), X_{2}=\left(\begin{array}{c}\lambda_{2} \\ 1\end{array}\right)$

set $P=\left(\begin{array}{cc}\lambda_{1} & \lambda_{2} \\ 1 & 1\end{array}\right)$, so $P^{-1}=\frac{1}{\lambda_{1}-\lambda_{2}}\left(\begin{array}{cc}1 & -\lambda_{2} \\ -1 & \lambda_{1}\end{array}\right)$

$A^{k}=P\left(\begin{array}{cc}\lambda_{1}{ }^{k} & 0 \\ 0 & \lambda_{2}{ }^{k}\end{array}\right) P^{-1}=\frac{1}{\lambda_{1}-\lambda_{2}}\left(\begin{array}{cc}\lambda_{1}{ }^{k+1}-\lambda_{2}{ }^{k+1} & \lambda_{1} \lambda_{2}{ }^{k+1}-\lambda_{2} \lambda_{1}{ }^{k+1} \\ \lambda_{1}{ }^{k}-\lambda_{2}{ }^{k} & \lambda_{1} \lambda_{2}{ }^{k}-\lambda_{2} \lambda_{1}{ }^{k}\end{array}\right)$

$\left(\begin{array}{c}F_{k+1} \\ F_{k}\end{array}\right)=A^{k}\left(\begin{array}{l}1 \\ 0\end{array}\right)=\frac{1}{\lambda_{1}-\lambda_{2}}\left(\begin{array}{c}\lambda_{1}{ }^{k+1}-\lambda_{2}{ }^{k+1} \\ \lambda_{1}{ }^{k}-\lambda_{2}{ }^{k}\end{array}\right)$

Get $F_{k}=\frac{1}{\sqrt{5}}\left[\left(\frac{1+\sqrt{5}}{2}\right)^{k}-\left(\frac{1-\sqrt{5}}{2}\right)^{k}\right]$

Led us through the above examples, the matrix eigenvalues in both modeling and application in differential equation, its main function is to matrix diagonalization, which can undertake high computation of matrix, thus the complexity of the simplified calculation. At the same time, is also due to matrix eigenvalue of the properties, allowing it to a wide range of applications. 


\section{References}

[1] Zhu Fengjuan :Eigenvalue and eigenvector research of inverse problem 。 Journal of binzhou college 2007.6 .

[2] Han Weihua: Matrix eigenvalue in the application of the linear differential equation .Teaching and science and technology , Shanghai, 1998,12(3,4)

[3] Zhang Xiaoxiang,Southeast university :Linear algebra modeling case assembly

[4] Lin Shaohua :Linear algebra .China water conservancy and hydropower press .2015,08 